\title{
Research on Key Technologies of Virtual Simulation Modeling for Switch Machine
}

\author{
Yang Zhao ${ }^{1, *}$, XiangWei Wang ${ }^{2}$ \\ ${ }^{1}$ School of Mechanical and Electrical Engineering, Huainan Normal University, Huainan 232038, China \\ ${ }^{2}$ Institute of Education, Huainan Normal University, Huainan 232038, China
}

\begin{abstract}
In order to improve the training effect, according to the mechanical structure of switch machine, combined with unity3D engine, a set of automatic learning system for switch machine based on virtual reality technology is designed.It realizes the guided teaching of switch equipment ,such as structure composition, three-dimensional model demonstration of action process and virtual disassembly. The system significantly improves the quality of training.
\end{abstract}

\section{Introduction}

Turnout is the switch equipment to complete the train from one track to another. The switch machine is the executive mechanism of the switch control system, which converts and locks the switch, reflects and supervises the position and state of the switch rail. The switch machine is one of the most important parts to ensure the safe and stable operation of trains, which is widely used in the field of rail transit. At present, the training methods related to the use and maintenance of various types of switch equipment are backward, and it is difficult to meet the needs of the trainees. This paper uses Unity3D engine, Pro/E, 3D Max, Photoshop and other technologies, combined with C\#script language to build a dynamic interactive and immersive training system for switch machine. The simulation modeling of switch machine can truly reproduce the field environment of switch equipment. Through theoretical study, virtual disassembly, and other practical operations, the trainees can truly experience the structural composition and maintenance operation of the switch machine. The application of the system in the training of rail transit company employees can help them better understand the working principle of the equipment, and then improve the operation and maintenance level of switch equipment.

\section{Structural analysis of turnout and switch equipment}

Turnout(shown in Figure 1), with high operation frequency, is the core component of the railway infrastructure. It is an essential device which moves the train from one track to another. The basic structure of turnout including switch rail, stock rail, straight closing rail, curved closing rail, wing rail, frog center and guard rail [1]. The switch has two movable switch rails, one is close to the stock rail, the other is separated. The switch equipment is installed at the switch rail, and the switch rail is dragged to turn the switch to the position or reverse position, so as to change the position of the switch.

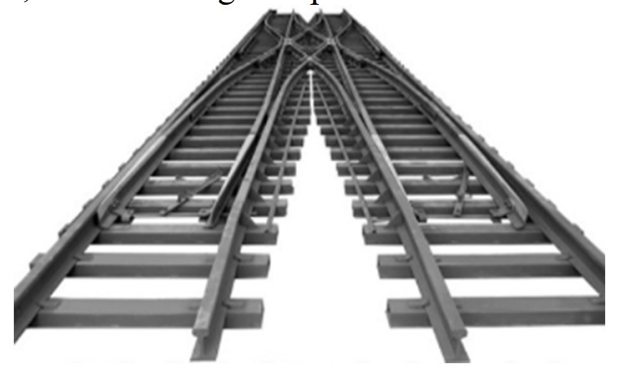

Fig. 1. Turnout system

ZD6 series DC electric switch machines are widely used in national railways, urban rail transit and local railways. ZD6 switch machine is a DC electric energy into the mechanical energy to push the rail switching device. The locking switch and the motor adopts a DC series excitation reversible motor, switch control using four wire switch, divided into start-up circuit and a switch indicating circuit. The starting circuit makes the electric switch for motor conversion switch, said circuit will switch position after conversion to reflect the signal tower, and gives the same site location representation (i.e., reverse), provide the conditions for the interlocking[2]. Switch start control has two kinds, one is route type switch control, to make the switch according to each route request will route switch to locate or reverse position; two is the independent operation of turnout in order to repair the experimental, the need for separate manipulation of turnout.

The composition of machine by the motor to see, a DC motor, speed reducer, friction coupling, start, automatic opening and closing device, main shaft, gear, rack block action (shown in Figure 2). DC electrical energy into mechanical energy; the speed reducer is the high speed motor in reducing friction; The switch is coupling is not in

*Corresponding author: 2841080064@qq.com 
place when rotating to increase friction; Start plate drives the checking column in the turnout at startup; Automatic shutter is in place after the start, the conversion switch connected to the corresponding position; Main shaft, gear, rack block with the vertical movement into the lateral force to drive switch. In addition to type ZD6-D, the E, F, $\mathrm{J}$, Gand other structures used in the field are approximately the same. Because the internal locking method is not suitable for the speed-up turnout, it is generally used for the side track of non speed-up section and speed-up section.

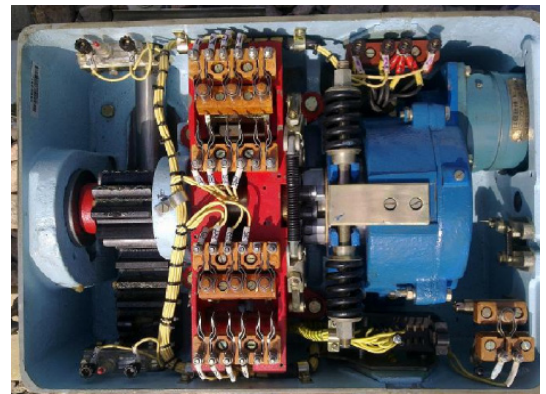

Fig. 2. Internal structure diagram of ZD6

Both S700K AC electric switch machine and ZYJ7 AC hydraulic switch machine used for speed-up switch adopt external locking mode, which is reliable and avoids highspeed impact of train. S700K switch machine is mainly composed of shell, power transmission mechanism, detection and locking mechanism, safety device and wiring interface. The structure of S700K AC electric switch machine is shown in Figure 3.

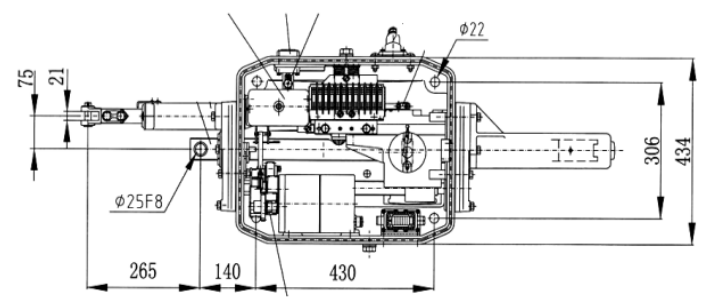

Fig. 3. mechanical structure of S700K switch machine

\section{Modeling process}

Due to the organization and management of the railway industry, the huge volume and high price of the signal equipment, the switch machine training generally adopts the teaching mode of teacher oriented, using the plane picture theory to teach the composition structure and working principle of the signal equipment. Students are in a passive position, it is difficult to transform the twodimensional knowledge into three-dimensional visual interactive objects, the teaching is lack of interactivity, and the satisfaction of teaching quality is poor.

Virtual reality technology is an important direction of simulation technology[3]. Through computer-generated simulation environment, the user can obtain the sense of controllability and immersion of the object in the simulation environment as the protagonist, and observe the internal structure of the model by means of humancomputer interaction. At present, it is widely used in military aerospace, interior design, 3D games and other fields. The automatic training system is built in a modular way with the research idea of "combination of virtual and real". It realizes the scene roaming, model display and interactive disassembly operation of switch equipment by using mouse and keyboard, and solves the limitation that students can not visit the railway line on the spot.

The construction and fidelity of three-dimensional model of switch machine is the core of the virtual simulation training system. This experimental system uses Unity3D as the development environment for VR model making and interaction. Firstly, through the design unit, physical measurement and national standard collection, we need to establish various technical parameters of the switch machine, so as to ensure the realistic effect of the simulation, and also as the data source of geometric modeling in Pro/E. Then built part model and assembly model are saved as *STL file, imported into 3D Max for physical modeling, and further completed the definition of constraint relationship and master-slave relationship. In order to enhance the realism of the environment in which the switch machine is used, the UV coordinates in 3D Max are used for texture mapping, such as the mapping of ballast and the environment where the switch machine is located[4]. The established model is stored in the model database, which can be used directly when similar components are needed next time to reduce the repetitive modeling work. Then import the exported *FBX file into Unity3D for real-time rendering of lighting effect, color and texture mapping.

\section{Optimization of switch machine model}

In order to ensure the smooth operation of the system under WinForm framework, it is necessary to use 3D modeling tools to optimize the original model[5]. The technical index requirements of point machine modeling are as follows:

(1) Response time and fluency. Due to the complex structure of the switch machine itself, there are rail, track bed, ballast and turnout in the virtual environment as trackside equipment, which have great restrictions on the real-time display speed of the switch machine. In the interaction process, the shorter the response time of the virtual scene, the better the system rendering fluency.

(2) Simplify the details of the model appropriately. The geometric model is simplified in detail. For example, the reducer can be simplified as an overall appearance model without refining the internal gear bearing combination. But for the accuracy of the important parts of the switch machine, it is necessary to ensure the display requirements of virtual disassembly.

(3) Ease of use in Engineering Teaching. The optimized model can be used in teaching examples to show the structure and virtual assembly process of switch machine. 


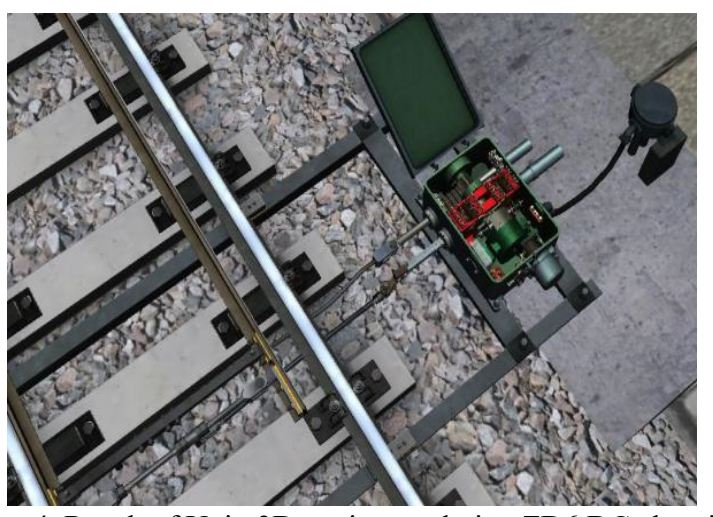

Fig. 4. Result of Unity3D engine rendering ZD6 DC electric switch machine(exclusion state)

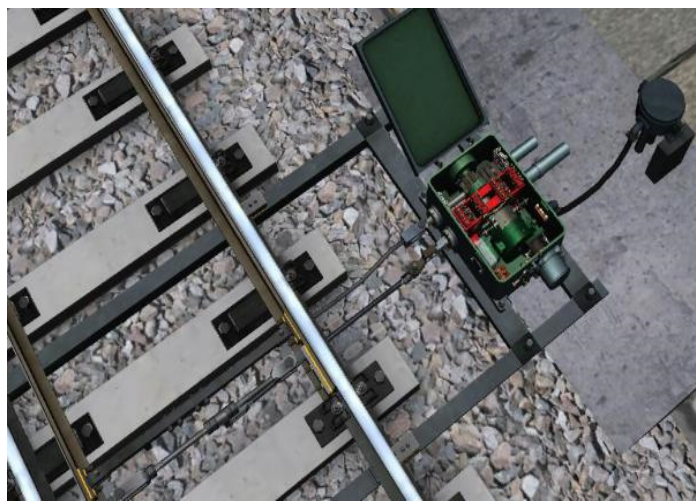

Fig. 5. Result of Unity3D engine rendering virtual models(close state)

Through figure 4 and figure 5, it shows the exclusion state and close state of ZD6 DC electric switch machine and switch rail. Figure 6 and 7 shows the model of S700K AC electric switch machine. The modeling of key components such as chassis, power transmission mechanism, detection and locking mechanism, safety device and other internal and external components are completed.

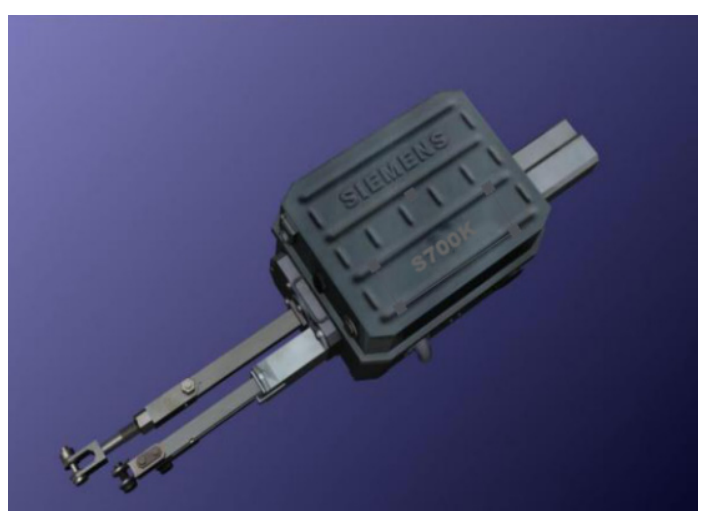

Fig. 6. Appearance of S700K

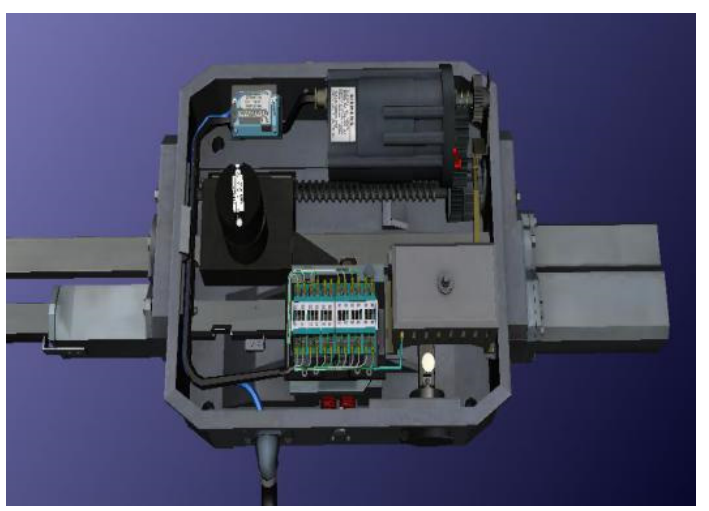

Fig. 7. Result of Unity3D engine rendering S700K (internal structure )

The program is written in Unity3D simulation software with C\# language to realize the interactive action of dragging and rotating the signal equipment. For example, drag the left mouse button to rotate the object. The basic principle is to convert the distance dragged by the mouse into the rotation angle of the object. Some of the core codes are as follows:

using UnityEngine;

using System.Collections;

public class NewBehaviourScript: MonoBehaviour

\{ private Vector3 startPoint; private Vector3 endPoint; private int disToAngle $=5$;

void Update () \{ if(Input.GetMouseButtonDown(0))

\{startPoint=Input.mousePosition;\} if(Input.GetMouseButtonUp(0))

\{endPoint=Input.mousePosition;

float $\mathrm{dx}=$ endPoint. $\mathrm{x}$-startPoint. $\mathrm{x}$;

float angle $=\mathrm{dx} / \mathrm{dis}$ ToAngle;

this.transform.localEulerAngles $=$ new

Vector3(0,angle, 0$)$; \}\}

\section{Conclusion}

Under the background of rapid development of urban rail transit and backward training mode of switch equipment, the system is designed by using virtual simulation technology combined with unity 3D. Through the vivid three-dimensional visualization interactive operation, the system has a realistic training environment. The system can improve the maintenance level of rail transit switch equipment, and has certain economic and social benefit.

\section{References}

1. X. Yang, X. Li, B. Ning, and T. Tang, "A survey on energy efficient train operation for urban rail transit," IEEE Transactions on Intelligent Transportation Systems, vol. 17, no. 1, pp. 2-13,(2016)

2. L.-H. Zhao and Q. Lu, "Method of turnout fault diagnosis based on grey correlation analysis," Journal of the China Railway Society, vol. 36, no. 2, pp. 6974, (2014)

3. Ouyang S G, Gang W, Yao J Y, et al. A Unity3D 
based interactive three-dimensional virtual practice platform for chemical engineering[J].Computer Applications in Engineering Education, (2017), 26(1)

4. Kim D, Ryu W, Lee S, et al. A Unity3D-based mobile fashion coordination system[J].International Journal of Advanced Media \& Communication, (2016), 6(1):86

5. Jiang Y, Xiao B, Yang B, et al. Study of Plant Animation Synthesis by Unity3D[M]// Computer and Computing Technologies in Agriculture VIII. (2015) 